\title{
Regulation of Klf16 by Double Strand RNA-binding Protein STAU1 in Adipocyte Differentiation in vitro
}

Ya Qun Guan ( $\nabla$ yaqunguan557@xjmu.edu.cn )

Xinjiang Medical University https://orcid.org/0000-0001-9164-1185

\section{Xuan Yu Meng}

Xinjiang Medical University

Xiao Di Liang

Xinjiang Medical University

Ting Ting Hu

Xinjiang Medical University

Nurbierye Nuermamati

Xinjiang Medical University

YI JIAO

Xinjiang Medical University

Xue Li Gong

Xinjiang Medical University

You Zhong Shen Xu

XinJiang medical university

Jia Le gao

Xinjiang Medical University

\section{Research}

Keywords: STAU1, 3T3-L1, adipocyte differentiation, post-transcriptional regulation, KIf16

Posted Date: July 16th, 2020

DOI: https://doi.org/10.21203/rs.3.rs-41788/v1

License: (c) (1) This work is licensed under a Creative Commons Attribution 4.0 International License.

Read Full License 


\section{Abstract}

Background: Adipogenesis is an essential process in organismal development and plays a significant role in adipose tissue homeostasis. Post-transcriptional regulation of gene expression plays a key role in adipogenesis and involves many RNA-binding proteins (RBPs). In mammals, Staufen1 (STAU1) is a conserved RBP(RNA Binding Protein )consisting of several dsRBP (double strand RNA). STAU1 plays an important role in the Stau1-mediated mRNA decay (SMD) pathway, which is related to adipocyte formation, myocyte development, and neural differentiation. Klf16 (Kruppel like transcription factor 16) is a negative regulator that inhibits adipocyte differentiation. AIM:This study was conducted to determine the role of KIf16 in adipocyte differentiation in the context of the SMD pathway.

Methods: 3T3-L1 cells were induced and cultured in vitro by cocktail method, Knockdown and Overexpression of STAU1 and KLF16. Then, adipocyte differentiation andexpression of adipogenicrelated genes (STAU1, KLF16, PPARY, and Lipin1) were measured by RT-qPCR and Western blot.RNA immunoprecipitation (RIP) method verified that STAU1 protein can bind to KLF16.

Results: The results revealed that STAU1 regulates KIf16 expression at the post-transcriptional level during the adipogenic differentiation of 3T3-L1 cells.STAU1 candirectly bind the 3'UTR of KIf16 mRNA. KIf16 mRNA was found to be degraded through the SMD pathway, thus promoting adipocyte differentiation.

Conclusions: In this study, the mechanism of adipocyte differentiation regulation at the posttranscriptional level is demonstrated, and KIf16 is shown as a substrate of the SMD pathway, thus providing new insights into adipogenesis.

\section{Introduction}

Obesity, as one of the main components of metabolic syndrome, is closely related to various diseases such as type 2 diabetes, dyslipidemia, hypertension, heart disease, and cancer. Abnormal differentiation and excessive proliferation of fat cells can lead to obesity [1,2]. Adipocyte differentiation is a complex process that is largely regulated by the activation of many transcription factors $[3,4]$. Among these, CCAAT/enhancer binding proteins (C/EBPs) and peroxisome proliferators (PPARY) are important transcription factors in adipocyte differentiation $[5,6]$.

Adipocyte differentiation is regulated mainly at the epigenetic, transcriptional, post-transcriptional, translational, and post-translational levels [7]. Recently, post-transcriptional regulation has been shown to play an important role in adipocyte differentiation [8]. Primary RNA, which is produced at the transcriptional level, must undergo a series of modifications to become mature RNA [9]. The 3'UTR region is an important part involved in its post-transcriptional regulation [10], which is in turn regulated by the trans-acting element, RNA-binding protein (RBP), and cis-acting sequence element. The 3'UTR can regulate the stability and degradation rate of mRNA [11], and can degrade useless mRNAs and some functional mRNAs [12]. The 3'UTR region of RBP plays an important role in post-transcriptional regulation 
during the growth and development of eukaryotic cells. For example, some mRNAs have local doublestranded structures that can be bound by RBPs [13], which can affect the stability of the mRNAs, resulting in their degradation [14-16]. STAU1 is highly expressed during the differentiation of 3T3-L1 adipocytes, and the number of adipocytes is increased after overexpression of STAU1. STAU1 is a kind of doublestranded RBP involved in the Stau1-mediated mRNA decay (SMD) pathway [13]. Thus, STAU1 recruits pUPF1 phosphate to activate the SMD pathway, which leads to degradation of its substrate. SMD substrates are characterized by a complex double chain structure in their 3'UTR regions and Alu sequence insertion [17]. STAU1 promotes adipocyte differentiation by reducing the stability of mRNAs that are negatively associated with adipocyte differentiation [18].

KIf16 is a member of the Kruppel like factor (KLF) family, which includes very important transcription regulators [19]. Klf16 plays an important role in adipogenesis and tumor growth [20, 21]. KLFs are transcription factors with a zinc finger structure. The typical structure shows $3 \mathrm{C} 2 \mathrm{H} 2$ zinc finger structures at the carboxyl terminal [22]. KIf16 is highly expressed in preadipocytes, but its level gradually decreases after adipocyte differentiation [23]. In this study, we examined the role of Klf16 in adipocyte differentiation in the context of the SMD pathway.

Our results provide new insight into the occurrence and development of obesity and dyslipidemia.

\section{Materials And Methods}

\section{Culture of 3T3-L1 cells}

Mouse 3T3-L1 fibroblasts were grown to the third-generation logarithmic growth phase and the cell suspension was adjusted to $1 \times 10^{5}$ cells/mLin DMEM containing $100 \mathrm{U} / \mathrm{mL}$ penicillin, $100 \mathrm{mg} / \mathrm{mL}$ streptomycin, $1 \%$ biotin and calcium pantothenate, and $10 \%$ fetal calf serum. The collected cells were seeded in a 6-well culture plate $(2 \mathrm{~mL} /$ well $)$ and cultured at $37{ }^{\circ} \mathrm{C}$ in an incubator with $5 \% \mathrm{CO}_{2}$. After $90 \%$ confluency and cell contact inhibition for $48 \mathrm{~h}$, lipogenic induction solution containing DMEM, insulin $(10 \mu \mathrm{g} / \mathrm{mL})$, 3-isobutyl-1-methylxanthine $(115 \mu \mathrm{g} / \mathrm{mL})$, and dexamethasone $(3.9 \mu \mathrm{g} / \mathrm{mL})$ was added. The cells were then cultured until they were completely differentiated.

\section{Oil Red 0 staining}

The cells (approximately $1 \times 10^{6}$ ) were washed three times with phosphate-buffered saline (PBS) and fixed in $4 \%$ paraformaldehyde (Sigma) for $2 \mathrm{~h}$ at room temperature. The cells were stained with $60 \%$ Oil Red O (Sigma) for 30 min and washed with PBS. For triglyceride quantification, Oil Red 0 was extracted with $100 \%$ isopropyl alcohol and the absorbance was measured at $500 \mathrm{~nm}$ (OD500). 


\section{Overexpression or knockdown of STAU1 and overexpression of Klf16}

Lipofectamine 3000, P3000 $2 \mu \mathrm{g} / \mu \mathrm{l}$ (Invitrogen), and $0.5-5 \mu \mathrm{g} / \mu \mathrm{l}$ of STAU1 shRNA plasmid were transfected into 3T3-L1 cells (approximately $5 \times 10^{5}$ ) and analyzed at $24 \mathrm{~h}$ after transfection. For STAU1 and KLF16 overexpression, 3T3-L1 cells were transfected with $1 \mu \mathrm{g}$ of STAU1 or KLF16-overexpression plasmid using Lipofectamine 3000 (Invitrogen) and were analyzed at $24 \mathrm{~h}$ after transfection. For the mRNA stability assay, 3T3-L1 cells (approximately $\left.5 \times 10^{5}\right)$ were treated with actinomycin D $(2.5 \mu \mathrm{g} / \mathrm{ml})$ for $1 \mathrm{~h}$, and cells harvested at $0,2,4$, and $6 \mathrm{~h}$ were examined by qPCR to determine the mRNA degradation rate.

\section{qRT-PCR}

Cells (approximately $5 \times 10^{5}$ ) seeded on $3.5-\mathrm{cm}$ dishes were used for RNA extraction with TRIzol reagent according to the manufacturer's instructions (Invitrogen, USA). RNA concentration and A260/A280 were measured by spectrophotometry to ensure the quality of RNA. cDNA was prepared using a Reverse Transcription Kit (Thermo). qRT-PCR was performed on a 7500 Real-Time PCR System (Applied Biosystems) using a SYBR-Green PCR Kit (Applied Biosystems). The values obtained were normalized to those of $28 \mathrm{~S}$ rRNA. Relative quantification was performed using the comparative cycle threshold $(\Delta \Delta \mathrm{Ct})$ method. The results were analyzed using an unpaired Student's t-test. P-values were calculated for samples from at least three independent experiments unless otherwise indicated. Primers used for qRTPCR were designed using primer design software (https://sg.idtdna.com/pages) and the sequences are listed in Table 1.

\section{Western blotting}

Whole-cell protein extracts were prepared from cells (approximately $1 \times 10^{6}$ ) lysed in RIPA buffer. Proteins were resolved by $8 \%$ SDS-PAGE and transferred onto nitrocellulose membranes (Thermo Scientific, USA). The immunoblots were incubated overnight at $4{ }^{\circ} \mathrm{C}$ with the following antibodies: anti-HSP90 (Proteintech), anti-STAU1 (Abcam), anti-KLF16 (Abcam), anti-LIPIN1 (Abcam), anti-UPF1 (Santa Cruz Biotechnology), and anti-pUPF1 (Millipore). The membranes were then incubated with horseradish peroxidase-conjugated secondary antibody (ZSGB-BIO, Beijing, China) at room temperature for $1 \mathrm{~h}$. Densitometric analysis was performed using Image Lab software (Bio-Rad).

\section{RNA immunoprecipitation (RIP)}

3T3-L1 cells (approximately $1 \times 10^{6}$ ) were added to RIP lysis buffer and RIP immunoprecipitation buffer, and incubated for $1 \mathrm{~h}$ at $4{ }^{\circ} \mathrm{C}$. Then, $50 \mu$ l Beads (17-701; Millipore) and $10 \mu \mathrm{l}$ anti-STAU1 (Abcam) were 
added and incubated overnight. The precipitates were washed 6 times with RIP washing buffer. Next, $150 \mu \mathrm{l}$ proteinase $\mathrm{K}$ solution (17-701; Millipore) was added and incubated at $55^{\circ} \mathrm{C}$ for $30 \mathrm{~min}$, followed by washing with RIP washing buffer. Finally, after adding $400 \mu \mathrm{l}$ of phenol: chloroform: isoprene mixture, RNA was extracted for PCR analysis.

\section{Chromatin IP (ChIP)}

Cells seeded on a $10-\mathrm{cm}$ dish were cross-linked with $275 \mu \mathrm{l}$ of $37 \%$ formaldehyde at room temperature for $10 \mathrm{~min}$, and $1 \mathrm{ml}$ glycine was then added and incubated at room temperature for $5 \mathrm{~min}$ to terminate the crosslinking. Cells were then lysed and sonicated to shear the chromatin to a manageable size. Next, $450 \mu \mathrm{l}$ of ChIP dilution buffer, $2.25 \mu \mathrm{l}$ protease inhibitor mixture, $20 \mu \mathrm{l}$ of fully suspended beads, and $10 \mu \mathrm{l}$ PPARy antibody were added to each IP, and incubated overnight at $4{ }^{\circ} \mathrm{C}$. DNA was extracted from the upper column of a 500- $\mu$ l Bind Reagent $A$, which was added after cleaning the magnetic beads, and PCR analysis was then performed.

\section{Photoactivatable ribonucleoside-enhanced crosslinking and immunoprecipitation (PAR-CLIP)}

When the 3T3-L1 cells reached 80\% confluence, they were incubated with $100 \mu \mathrm{M}$ 4-thiouridine (SU) for $16 \mathrm{~h}$, washed with PBS, and irradiated with $150 \mathrm{~mJ} / \mathrm{cm}^{2}, 356 \mathrm{~nm}$ ultraviolet light in a crosslinker for crosslinking RNA to Stau1. Alternatively, samples were harvested and lysed in an equivalent of three pellet volumes of NP-40 lysis buffer. Cell lysates were then treated with $1 \mathrm{U} / \mu \mathrm{l}$ of RNase T1 (Thermo), and endogenous Stau1 protein was immunoprecipitated using polyclonal anti-Stau1 antibody (Abcam) bound to beads. RNA in the immunoprecipitates was further trimmed with $100 \mathrm{U} / \mathrm{ml}$ RNase T1. Beads were washed in lysis buffer, and DNA and proteins were removed by digestion with DNase I (Thermo) and $0.2 \mathrm{mg} / \mathrm{ml}$ proteinase $\mathrm{K}$ in proteinase $\mathrm{K}$ buffer (Thermo), respectively. RNA was recovered by acidic phenol/chloroform extraction and ethanol precipitation, and was used for qPCR analysis. Primers for each section of the KIf16 3'UTR region were designed and the exact binding sites of STAU1 and KIf16 were detected by qPCR.

\section{Statistical analysis}

The data are presented as the mean \pm standard error of the mean (SEM) from three independent experiments, and three replicates were evaluated for each experiment. Statistical differences were measured by a Student's t-test or two-way ANOVA with Bonferroni's correction for multiple comparisons, when appropriate. $\mathrm{P}<0.05$ was considered to indicate a statistically significant difference. Data analysis was performed using GraphPad Prism 5.0 software (GraphPad, San Diego, CA, USA).

\section{Results}




\section{Expression of STAU1, Klf16, and PPARY in adipogenesis}

In order to verify the expression of STAU1, KIf16, and PPARY during the induction and differentiation of 3T3-L1 cells, 3T3-L1 preadipocytes were induced using the cocktail method and were analyzed by Oil Red 0 staining on day $0,2,4,6$, and 8 of differentiation. Two days after cell differentiation, stained red lipid droplets were observed, their number was gradually increased, and they accumulated on days 4,6 , and 8 (Fig. 1a, b). After induction, the mRNA and protein levels of STAU1 and KIf16 at days 0, 2, 4, 6, and 8 were determined. After 4 days of induction, the protein (Fig. 1c) and mRNA (Fig. 1d) levels of STAU1 and PPARy were significantly increased $(P<0.05)$, whereas the expression of Klf16 protein (Fig. 1C) and mRNA (Fig. 1d) was significantly decreased $(P<0.05)$. It was observed that in mature 3T3-L1 adipocytes, STAU1 and PPARY were highly expressed, whereas KIf16 showed low expression.

\section{Overexpression of STAU1 reduces KLF16 expression in adipogenesis}

We then examined whether STAU1 overexpression affected the mRNA and protein levels of KIf16 and key SMD factors. 3T3-L1 pre-adipocytes were induced to differentiate into mature adipocytes, and transfected with a STAU1 overexpression plasmid. The mRNA and protein levels of STAU1, KIf16, pUPF1, and UPF1 were then measured at 0, 1, 2, 3, and 4 days. The results of Oil Red 0 staining showed that lipid droplets were more obvious in the STAU1 overexpression group than in the control group (Fig. 2a, b). The mRNA and protein levels of STAU1 indicated that the STAU1 overexpression efficiency was $65 \%$ (Fig. 2c, d). Compared to that in the control group, the mRNA expression of KLF16 in the STAU1 overexpression group showed no significant difference (Fig. 2C), whereas its protein expression was significantly reduced $(P<0.05)$ (Fig. 2d). Expression of the key SMD pathway factor, pUPF1, was significantly increased at the protein and mRNA levels $(P<0.05)$, whereas UPF1 showed no difference (Fig. 2d). It was thus observed that STAU1 overexpression did not affect the mRNA expression of KLF16, but affected the protein expression of KLF16, STAU1, and pUPF1.

\section{STAU1 knockdown increases KLF16 expression in adipogenesis}

To further examine the mRNA and protein levels of KIf16, pUPF1, and UPF1, 3T3-L1 cells were transfected with Stau1 shRNA1 and Stau1 shRNA2. The mRNA and protein levels of STAU1, KIf16, pUPF1, and UPF1 were measured at $0,1,2,3$, and 4 days. The results of Oil Red 0 staining showed that the STAU1 knockdown group had less lipid droplets than the control group (Fig. 3a, b). The STAU1 knockdown efficiency was about $65 \%$, and there was no significant difference in the KIf16 mRNA levels between the STAU1 knockdown group and the control group (Fig. 3c). However, compared to that in the control group, the protein level of KIf16 in the STAU1 knockdown group was significantly increased $(P<0.05)$, whereas the STAU1 and pUPF1 levels were significantly decreased $(P<0.05)$, and the total UPF1 levels remained 
unchanged (Fig. 3d). The above results jointly indicate that STAU1 affects the stability of KIf16 mRNA at the post-transcriptional level, thereby affecting the production of fat and promoting adipocyte differentiation.

\section{STAU1 specifically binds the 3' UTR of KIf16 mRNA}

In order to further study the binding sites between STAU1 and KIf16 RNA during the differentiation of 3T3L1 adipocytes, we used RNA co-precipitation experiments to verify the binding of STAU1 and KIf16 mRNA, and PCR confirmed that STAU1 and KIf16 bind at D2 and D4 (Fig. 4a). PAR-CLIP experiments were performed on 3T3-L1 cells to verify the binding of STAU1 to the KLF16 3'UTR. Ten pairs of primers were designed according to the length of the fragment. Each primer pair represents the length of a fragment in the 3'UTR region (Fig. 4b), and qPCR analysis was conducted. We found that KIf16 mRNA contained at least one identified region and was bound by STAU1. ChIP experiments proved that PPARY could combine with the promoter region of Klf16 and that its binding ability is increasingly strong (Fig. 4c).

After transfection of 3T3-L1 pre-adipocytes with STAU1 overexpression plasmid for $24 \mathrm{~h}$, the cells were treated with actinomycin D for $1 \mathrm{~h}$ and then collected at 0, 2, 4, and $6 \mathrm{~h}$. Klf16 mRNA expression was determined by qPCR. The degradation rate of KIf16 mRNA was significantly accelerated after STAU1 overexpression compared to that in the control group $(P<0.05)$. STAU1 was confirmed to affect the KLF16 mRNA degradation rate (Fig. 4d). These data provide some evidence that STAU1 could specifically bind to KLF16 3'UTR and affect its mRNA degradation rate.

\section{KIf16 regulates Lipin1 to affect adipogenesis}

In order to study the downstream target of Klf16, Oil Red 0 staining was performed after Klf16 overexpression, and morphological changes were observed. KIf16 overexpression resulted in significantly reduced lipid droplets compared to the control group $(P<0.05)$ (Fig. 5a, b). Compared to that in the control group, the mRNA and protein expression of Lipin1 in the KIf16-overexpression group was significantly downregulated as detected by qPCR and western blotting, respectively $(P<0.5)$ (Fig. $5 c$, d). Intracellular triglyceride content was decreased during 3T3-L1 differentiation compared with that in the control group after overexpression of KLF16 (Fig. 5e). This suggests that Klf16 inhibits the gene expression of Lipin1, thereby inhibiting adipogenesis.

\section{Discussion}

Obesity is defined as the excessive accumulation and/or abnormal distribution of fat in the body. It is a chronic metabolic disease caused by the interaction of genetic and environmental factors [1,24]. A large number of studies have shown that STAU1 can regulate cell growth and nerve differentiation [25]. However, little is known about the post-transcriptional regulation of adipogenesis by STAU1. STAU1 can directly bind to the 3'UTR of KIf16 mRNA (Fig. 4), leading to mRNA decay, which results in increased 
expression of LIPIN1 mRNA and protein, consequently promoting the formation of triglycerides and improving adipogenesis (Fig. 5). KLF16 is a negative regulator of adipocyte differentiation. KLF16 expression inhibits the synthesis of fatty acids and triglycerides, thus inhibiting the differentiation of adipocytes. It is known that the regulation of mRNA stability after transcription in the 3'UTR region is mainly controlled by sequences containing ARE (AU-richelement)elements [26]. In addition to microRNAmediated mRNA decay [27] and NMD (nonsense-mediated mRNA decay, [nonsense-mediated mRNA degradation [28], it was first proposed that STAU1 could bind to the 3'UTR region of KIf16 and degrade its mRNA at the post-transcriptional level in the SMD pathway. In addition to the many biological functions involving STAU1, there is growing evidence that STAU1 and other IncRNAs can also affect metabolic homeostasis by influencing target genes. STAU1 is also reportedly involved in lipid formation by influencing KLF2 [13].

In 3T3-L1 cells, mRNAs with a specific secondary structure and binding to STAU1 were screened by RIP. Among these mRNAs, Klf2, KIf16, KIf4, and KIf14 all had high peaks for STAU1 binding and are known to play an important role in early adipocyte differentiation. After STAU1 knockdown and overexpression, we found that the protein level of KIf16, a novel negative regulator of adipocyte differentiation, had changed (Fig. 4). We thus assumed that KIf16 is a new substrate of the SMD pathway. The PAR-CLIP experiment showed that STAU1 had a high binding peak at the 853-981 bases (128 bases) of the KIf16 mRNA 3'UTR, indicating that this segment had a binding site for STAU1, but studying its exact site more precisely is required. ChIP validated the effect of PPARY on KLF16 promoter activity (Fig. 4). In 3T3-L1 cells, the expression of PPARy was increased with adipocyte differentiation. ChIP results showed that PPARY and KIf 16 exhibited a stronger binding capacity, demonstrating that increasingly more KIf 16 was transcribed. However, the mRNA expression of Klf16 was decreased during adipocyte differentiation, indicating that the degradation of KIf16 during adipocyte differentiation was regulated at the posttranscriptional level. Klf16 overexpression resulted in a decrease in triglyceride levels and reduced the mRNA and protein expression of Lipin1, a phosphatidyl phosphatase necessary for the biosynthesis of triglycerides (Fig. 5). Lipin1 can assist the transcription of PPARy and regulate lipid metabolism gene expression.

This study also revealed that the expression of C/EBPa mRNA was significantly decreased compared to that in the control group after Klf16 overexpression. C/EBPa is an important transcription factor, and KIf16 has a lower expression in late adipocyte differentiation. Previous studies have found that KIf16 suppresses the PPARy promoter to inhibit its expression. PPARy promotes the expression of C/EBPa. Thus, Klf16 inhibits the expression of PPARY and C/EBPa.

The mechanism of STAU1 function in the process of adipocyte differentiation has been reported. After the knockout of STAU1, the differentiation of adipocytes was obviously weakened. The late target gene of STAU1, KLF16, is involved in the formation of adipocytes; this provides a clearer molecular mechanism for clinical applications and cellular and molecular basis for targeted gene therapy for the treatment of obesity. However, this study only draws conclusions from the cellular level, lacking validation by in vivo data. 
The role of KLF16 in adipocyte differentiation has been reported, but the involvement of the SMD pathway in neuronal differentiation, muscle generation, and cancer development has not been clearly reported and still requires further study.

\section{Declarations}

\section{Conflict of Interest:}

The authors declare that they have no conflict of interest.

\section{Funding:}

This study was funded by the National Natural Science Foundation of China [grant numbers 81760162 , 81660154] and 2017 University Research Program [grant number XJEDU2017M016]. The funding agency had no role in the study design, the collection, analysis, or interpretation of data, the writing of the report, or the decision to submit the article for publication.

\section{References}

1. S.D. Rosa, B. Arcidiacono, E. Chiefari, et al., Type 2 diabetes mellitus and cardiovascular disease: genetic and epigenetic links, Front. Endocrinol. 9 (2018) 2. https://doi.org/10.3389/fendo.2018.00002

2. J.O. Ebbert, M.D. Jensen, Fat depots, free fatty acids, and dyslipidemia, Nutrients. 5 (2013) 498-508. https://doi.org/10.3390/nu5020498.

3. K. Yu, D. Mo, W. Ming, et al., Activating transcription factor 4 regulates adipocyte differentiation via altering the coordinate expression of CCATT/enhancer binding protein $\beta$ and peroxisome proliferatoractivated receptor $\gamma$, FEBS J. 281 (2014) 2399-2409. https://doi.org/10.1111/febs.12792.

4. A.T. Ali, W.E. Hochfeld, R. Myburgh, et al., Adipocyte and adipogenesis, Eur. J. Cell Biol. 92(2013)229236. https://doi.org/10.1016/j.ejcb.2013.06.001.

5. J.N. Ho, J.W. Choi, W.C. Lim, et al., Kefir inhibits 3T3-L1 adipocyte differentiation through downregulation of adipogenic transcription factor expression,J. Sci. Food Agric. 93 (2013) 485-490. https://doi.org/10.1002/jsfa.5792.

6. L. Guo, X. Li, Q.Q. Tang, Transcriptional regulation of adipocyte differentiation: a central role for CCAAT/enhancer-binding protein (C/EBP) $\beta$, J. Biol. Chem. 290 (2015) 755-761. https://doi.org/10.1074/jbc.R114.619957.

7. K. Fujimori, Y. Urade, Transcriptional regulation in adipogenesis through ppary-dependent and independent mechanisms by prostaglandins, in: E. Miyamoto-Sato, H. Ohashi, H. Sasaki, J. 
Nishikawa, H. Yanagawa (Eds.), Transcription Factor Regulatory Networks. Methods in Molecular Biology (Methods and Protocols), Humana Press, New York, 2014, vol. 1164.

8. Y.J. Hu, H. Belaghzal, W.Y. Hsiao, et al., Transcriptional and post-transcriptional control of adipocyte differentiation by Jumonji domain-containing protein 6, Nucleic Acids Res. 43 (2015) 7790-7804. https://doi.org/10.1093/nar/gkv645.

9. C. Mayr, Evolution and biological roles of alternative 3'UTRs, Trends Cell Biol. 26 (2016) 227-237. https://doi.org/10.1016/j.tcb.2015.10.012.

10. M.Kim, N. Kogan, F.J. Slack, Cis-acting elements in its 3' UTR mediate post-transcriptional regulation of KRAS, Oncotarget. 7 (2016) 11770-11784. https://doi.org/10.18632/oncotarget.7599.

11. Y. Zhang, M.S. Sachs, Control of mRNA stability in fungi by NMD, EJC and CBC factors through 3'UTR introns, Genetics. 200 (2015) 1133-1148. https://doi.org/10.1534/genetics.115.176743.

12. E. Park, L.E. Maquat, Staufen-mediated mRNA decay, Wiley Interdiscip. Rev. RNA. 4 (2013) 423-435. https://doi.org/10.1002/wrna.1168.

13. H. Cho, K.M. Kim, S. Han, et al., Staufen1-mediated mRNA decay functions in adipogenesis, Mol. Cell. 46 (2012) 495-506. https://doi.org/10.1016/j.molcel.2012.03.009.

14. L. Wurth, F. Gebauer, RNA-binding proteins, multifaceted translational regulators in cancer, Biochim. Biophys. Acta Gene Regul. Mech. 1849 (2015) 881-886. https://doi.org/10.1016/j.bbagrm.2014.10.001.

15. C. Shi, S. Wang, T. Zhou, et al., Post-transcriptional regulation tends to attenuate the mRNA noise and to increase the mRNA gain, Physical Biol. 12 (2015) 056002. https://doi.org/10.1088/14783975/12/5/056002.

16. A. Radhakrishnan, R. Green, Connections underlying translation and mRNA stability, J. Mol. Biol. 428 (2016) 3558-3564. https://doi.org/10.1016/j.jmb.2016.05.025.

17. C. Gong, L.E. Maquat, IncRNAs transactivate STAU1-mediated mRNA decay by duplexing with $3^{\prime}$ UTRs via Alu elements, Nature. 470 (2011) 284-288. https://doi.org/10.1038/nature09701.

18. M.Y. Kim, J. Park, J.J. Lee, et al., Staufen1-mediated mRNA decay induces Requiem mRNA decay through binding of Staufen1 to the Requiem 3'UTR, Nucleic Acids Res. 42 (2014) 6999-7011. https://doi.org/10.1093/nar/gku388.

19. C.W. Brey, M.P. Nelder, T. Hailemariam, et al., Krüppel-like family of transcription factors: an emerging new frontier in fat biology, Int J Biol Sci. 5 (2009) 622-636.

20. N.M. Pollak, M. Hoffman, I.J. Goldberg, et al., Krüppel-like Factors: crippling and uncrippling metabolic pathways, JACC Basic Transl. Sci. 3 (2018) 132-156. https://doi.org/10.1016/j.jacbts.2017.09.001.

21. P. Ma, C.Q. Sun, Y.F. Wang, et al., KLF16 promotes proliferation in gastric cancer cells via regulating p21 and CDK4, Am. J. Transl. Res. 9 (2017) 3027-3036.

22. R. Pearson, J. Fleetwood, S. Eaton, et al., Kruppel-like transcription factors: a functional family, Int. J. Biochem. Cell Biol. 40 (2008) 1996-2001. https://doi.org/10.1016/j.biocel.2007.07.018. 
23. M.K. Jang, S. Lee, M.H. Jung, RNA-Seq analysis reveals a negative role of KLF16 in adipogenesis, PLoS One. 11 (2016) e0162238. https://doi.org/10.1371/journal.pone.0162238.

24. T.R. Gaillard, The metabolic syndrome and its components in African-American women: emerging trends and implications, Front. Endocrinol. 8 (2018) 383. https://doi.org/10.3389/fendo.2017.00383.

25. B.S. Moon, J. Bai, M. Cai, et al., Kruppel-like factor 4-dependent Staufen1-mediated mRNA decay regulates cortical neurogenesis, Nat. Commun. 9 (2018) 401. https://doi.org/10.1038/s41467-01702720-9.

26. G. Stoecklin, S. Hahn, C. Moroni, Functional hierarchy of AUUUA motifs in mediating rapid interleukin3 mRNA decay, J. Biol. Chem. 269 (1994) 28591-28597.

27. H.O. Iwakawa, Y. Tomari, The functions of microRNAs: mRNA decay and translational repression, Trends Cell Biol. 25 (2015) 651-665. https://doi.org/10.1016/j.tcb.2015.07.011.

28. T. Kurosaki, W. Li, M. Hoque,et al., A post-translational regulatory switch on UPF1 controls targeted mRNA degradation, Genes Dev. 28 (2014) 1900-1901. https://doi.org/10.1101/gad.245506.114.

\section{Figures}

a

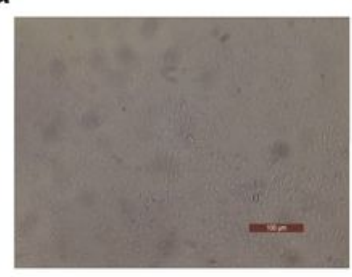

0

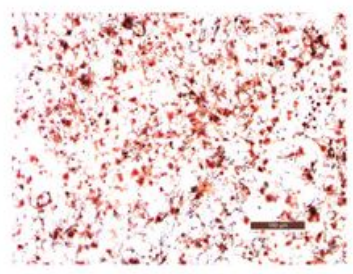

2

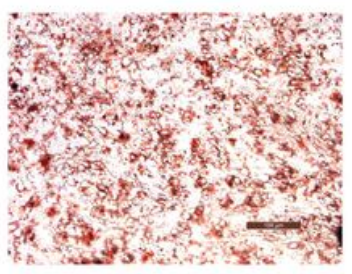

4

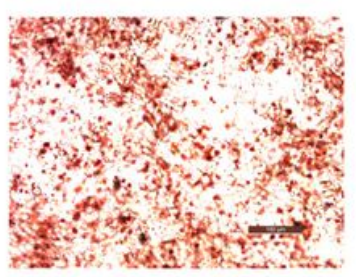

6

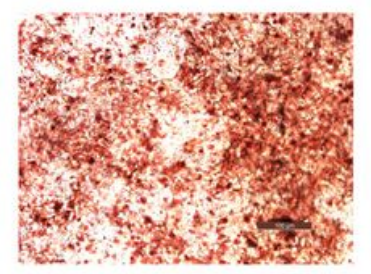

8

Day

b
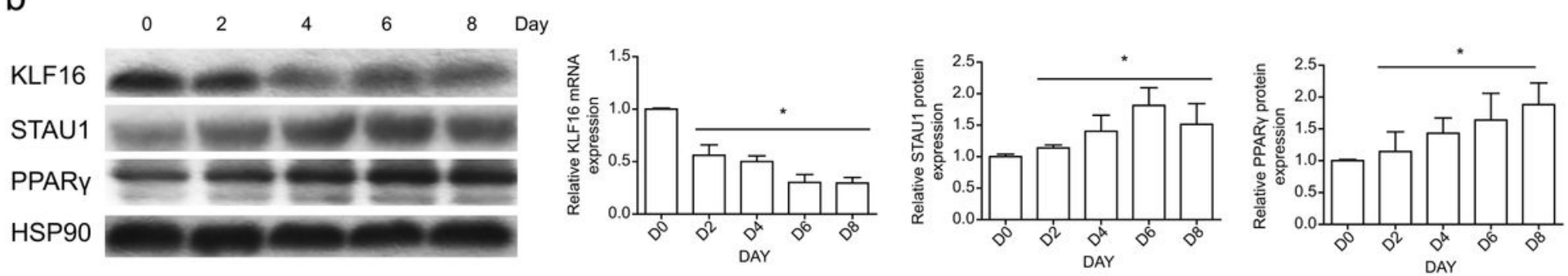

C
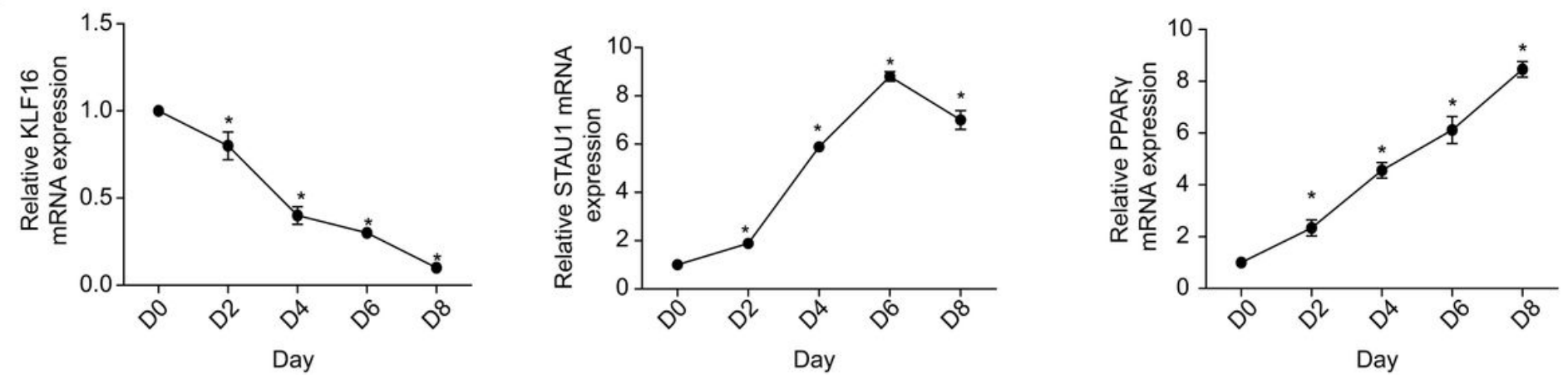

Figure 1 
Expression of STAU1, KIf16, and PPAR in differentiation-induced 3|3-L1 cells Representative Oil Red 0 staining images on days $0,2,4,6$, and 8 after adipocyte differentiation in 3T3-L1 cells (a-b). mRNA and protein levels of STAU1 and PPARy were increased on days 2, 4, 6, and 8 compared to day 0 of differentiation in 3T3-L1 cells. Decreased mRNA and protein expression of KLF16 was seen at days 0, 2, 4,6 , and 8 (c-d) The results are presented as the mean $\pm S D\left({ }^{\star} P<0.05\right.$, compared with day 0 , which was defined as undifferentiated 3T3-L1)

a

Control

STAU1

over

expression

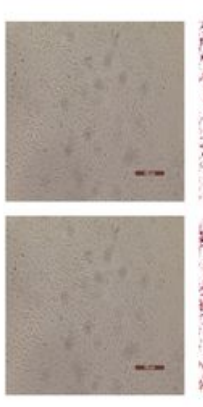

0

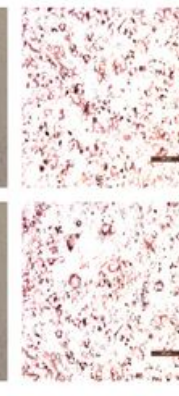

1

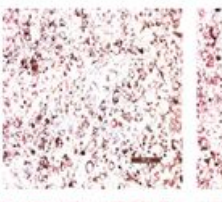

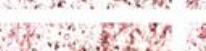
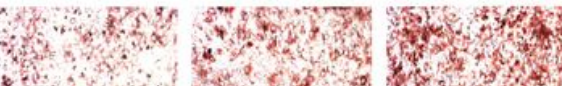

\&

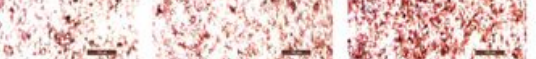

2

3

STAU1 over expression

Control

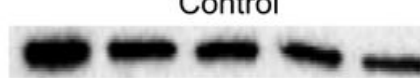

KLF16

STAU1

pUPF1

UPF1

HSP90
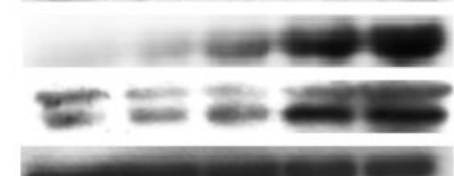

$$
\text { c }
$$

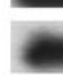

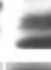

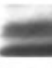

4
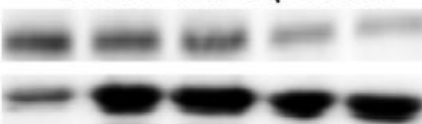

$$
\begin{array}{lllllllllll}
0 & 1 & 2 & 3 & 4 & 0 & 1 & 2 & 3 & 4 & \text { Day }
\end{array}
$$
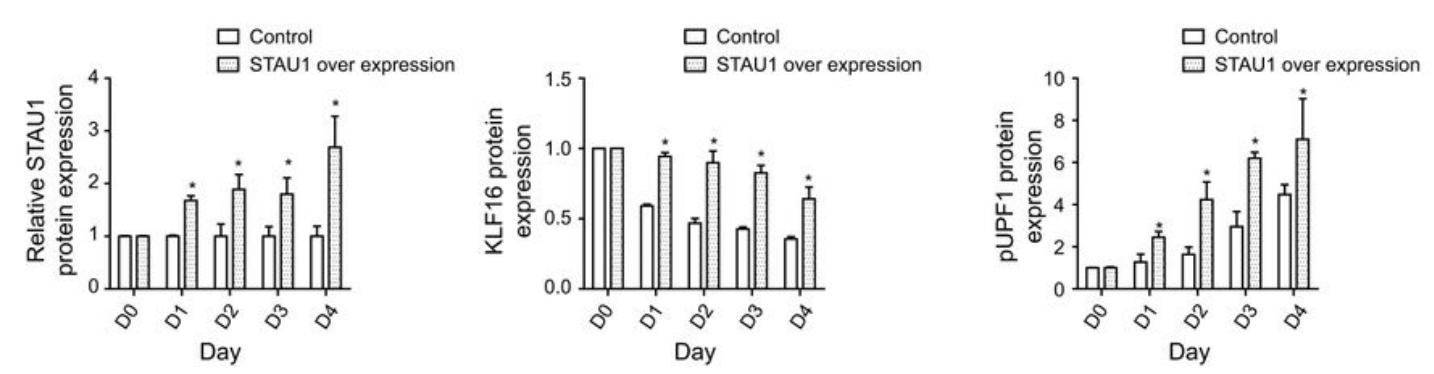

b

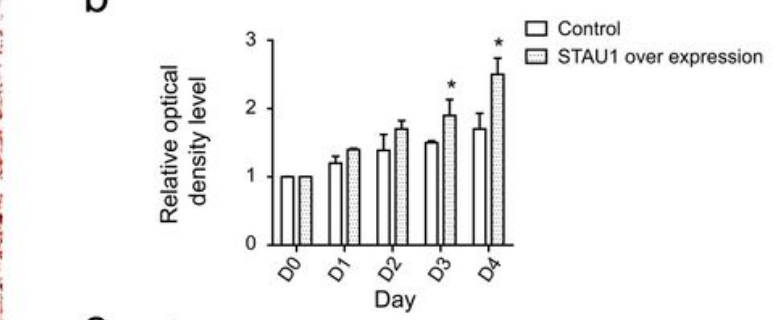

\section{Day}
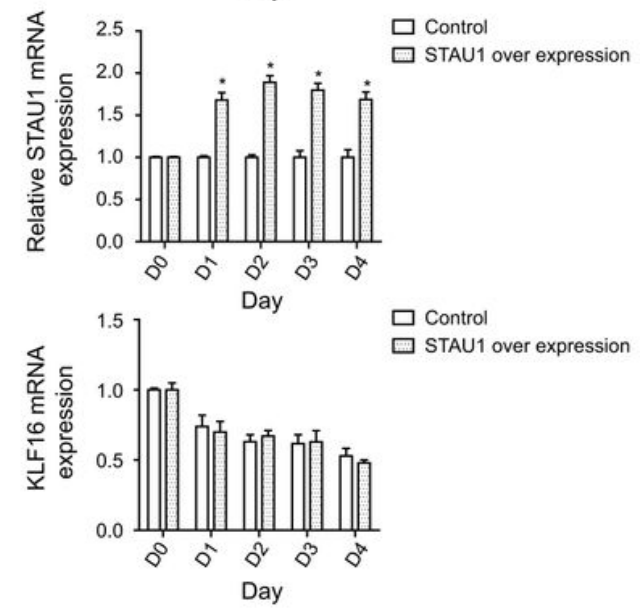

\section{Figure 2}

Effect of STAU1 overexpression on KIf16 expression Representative Oil Red O staining images of differentiation-induced adipocytes transfected with STAU1-overexpression plasmid on days 1, 2, 3, and 4 $(a-b)$. The effects of STAU1 overexpression on the mRNA (c) and protein levels (d) of STAU1, pUPF1, UPF1, and KLF16 compared to the control groups on days 0, 1, 2, 3, and 4 of 3T3-L1 adipocyte differentiation. ( ${ }^{*} \mathrm{P}<0.05$, compared with day 0 , which was defined as undifferentiated 3T3-L1) 
a

Control

STAU1 shRNA1

STAU1 ShRNA2

0

Control

2

d

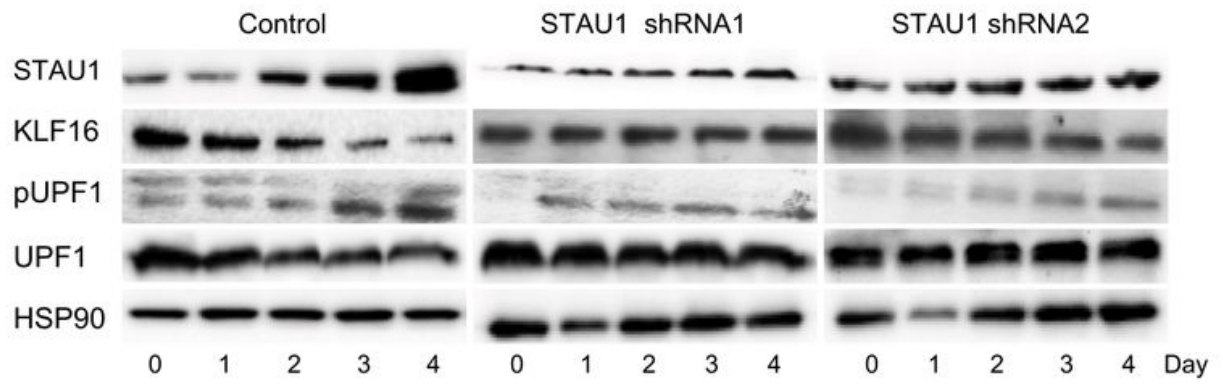

Day
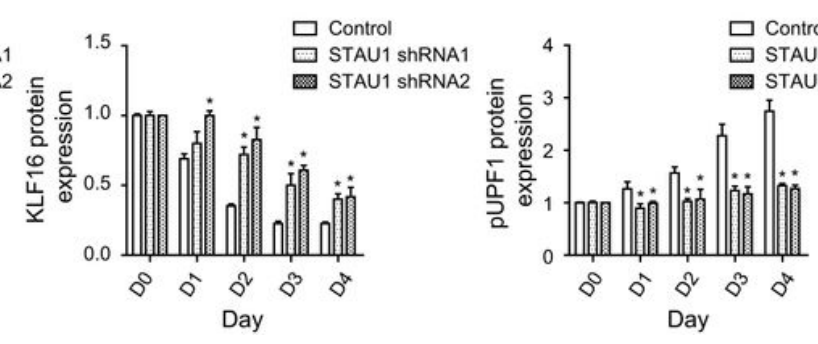

b

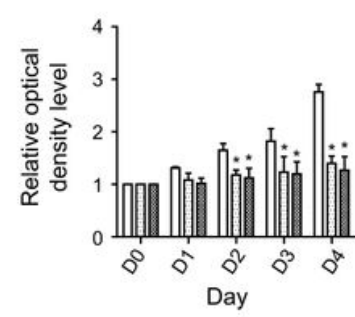

C

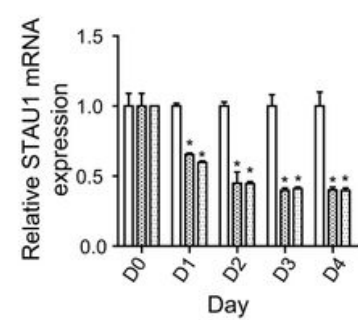

$\square$ Control

STAU1 ShRNA1 STAU1 ShRNA2

$\square$ Control

STAU1 ShRNA1

STAU1 shRNA2

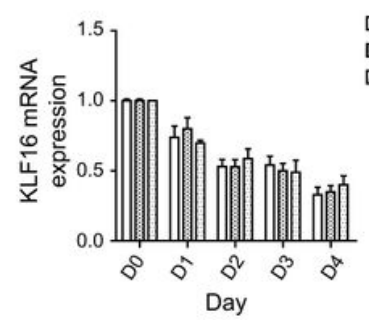

$\square$ Control

STAU1 ShRNA1

STAU1 ShRNA2
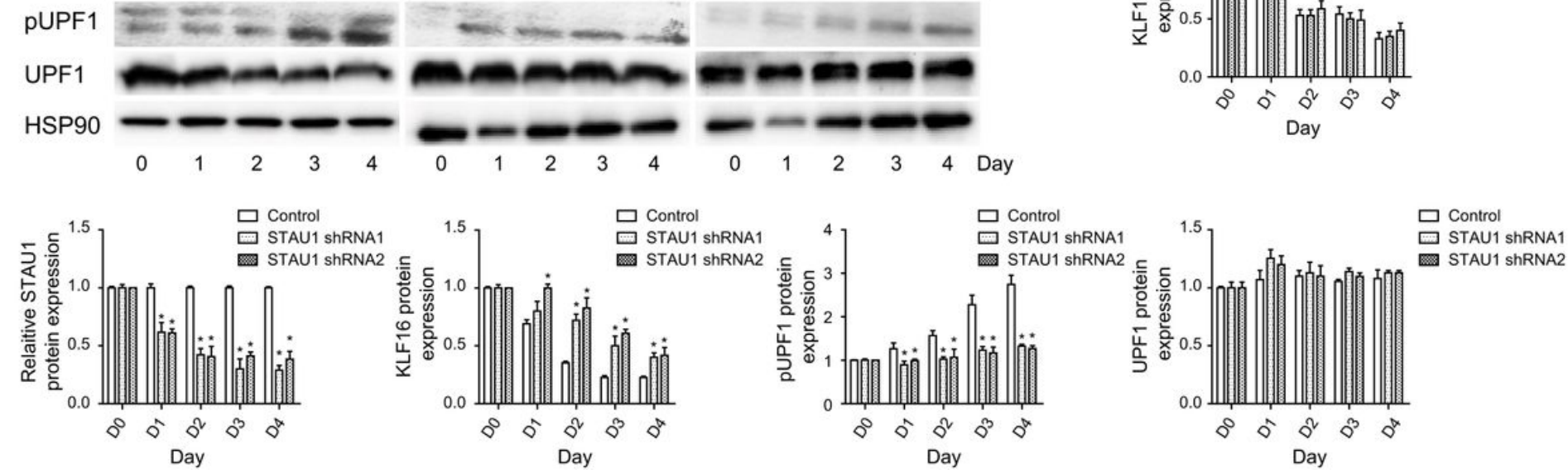

Figure 3

Expression level of KIf16 after STAU1 knockdown Representative Oil Red 0 staining images of differentiation-induced adipocytes transfected with STAU1 shRNA1and STAU1 shRNA2plasmidsondays $1,2,3$, and $4(a-b)$. The effects of STAU1 shRNA1 and STAU1 shRNA2 on the mRNA (c) and protein levels (d) of STAU1, KLF16, pUPF1, and UPF1 compared to the control group on days 1,2, 3, and 4 of 3T3-L1 adipocyte differentiation. ( ${ }^{\star} \mathrm{P}<0.05$, compared with day 0 , which was defined as undifferentiated 3T3-L1) 
a

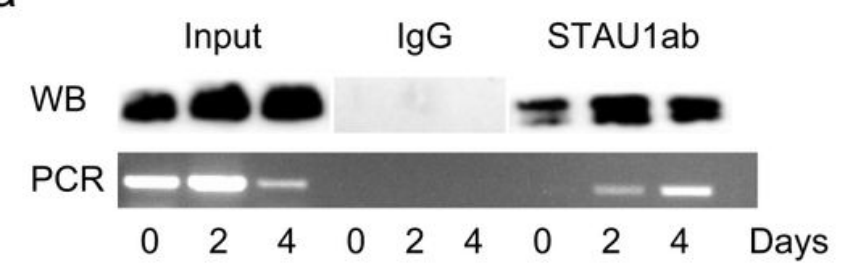

C

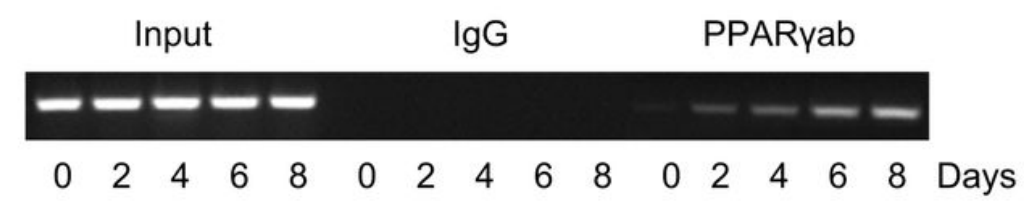

d b

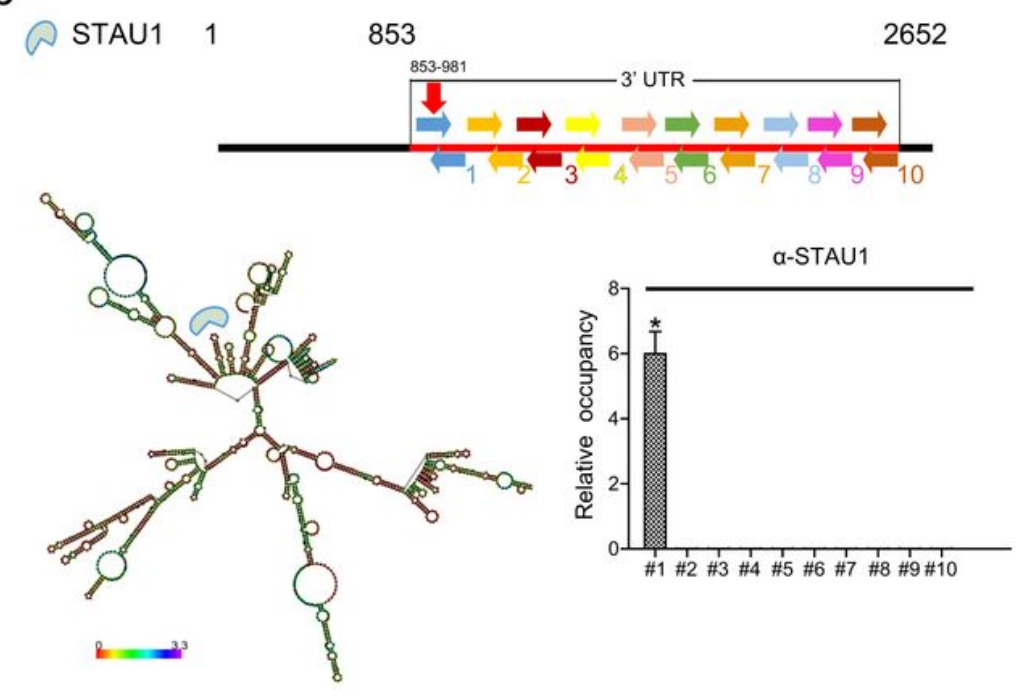

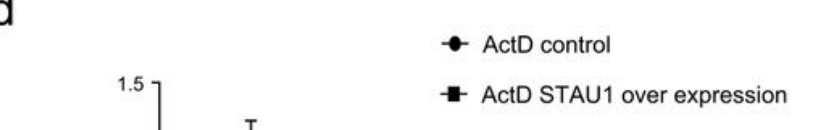

\section{Figure 4}

STAU1 specifically binds the 3'UTR of KIf16 mRNA RIP analysis of STAU1 and KLF16 mRNA was performed using IgG as a construct and an anti-STAU1 antibody. Quantification of KLF16 mRNA by PCR on days 0,2 , and 4 during the differentiation of 3T3-L1 adipocytes (a). The PAR-CLIP assay was performed in 3T3-L1 cells. The KLF16 3'UTR was divided into ten segments and primers were designed. The binding sites of STAU1 and KLF16 3'UTR were verified by qPCR. (b). ChIP analysis of STAU1 and KLF16 mRNA was performed using IgG as a control, INPUT as a positive control, and an anti-STAU1 antibody. The binding ability of KLF16 and PPARy was examined by PCR on days 0, 2, 4, 6, and 8 during the differentiation of 3T3-L1 adipocytes(c).3T3-L1 were induced for differentiation over $72 \mathrm{~h}$ with transfection for STAU1overexpression for $24 \mathrm{~h}$, and actinomycin D for 2, 4, and $6 \mathrm{~h}$. Levels ofKLF16mRNA expression were determined by qRT-PCR. The results are presented as the mean \pm SEM $\left({ }^{\star} P<0.05\right.$, compared with control) (d) 
a

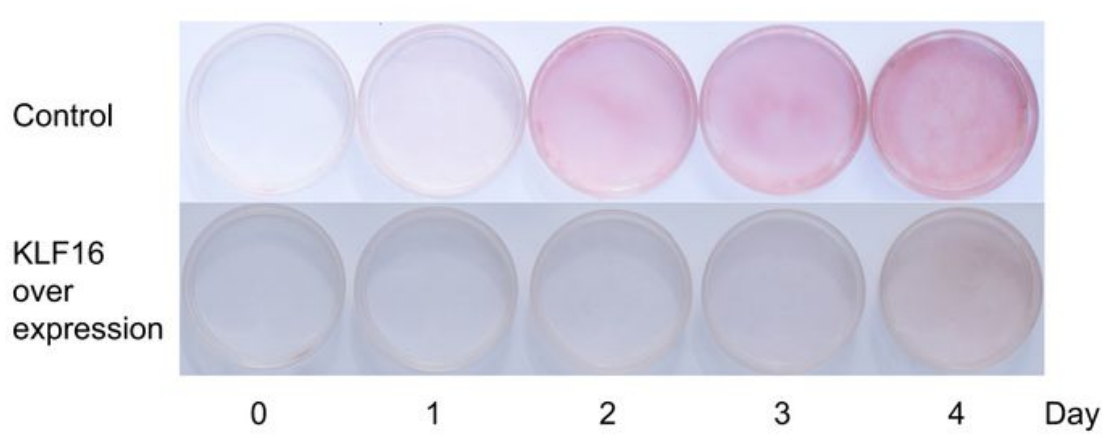

d
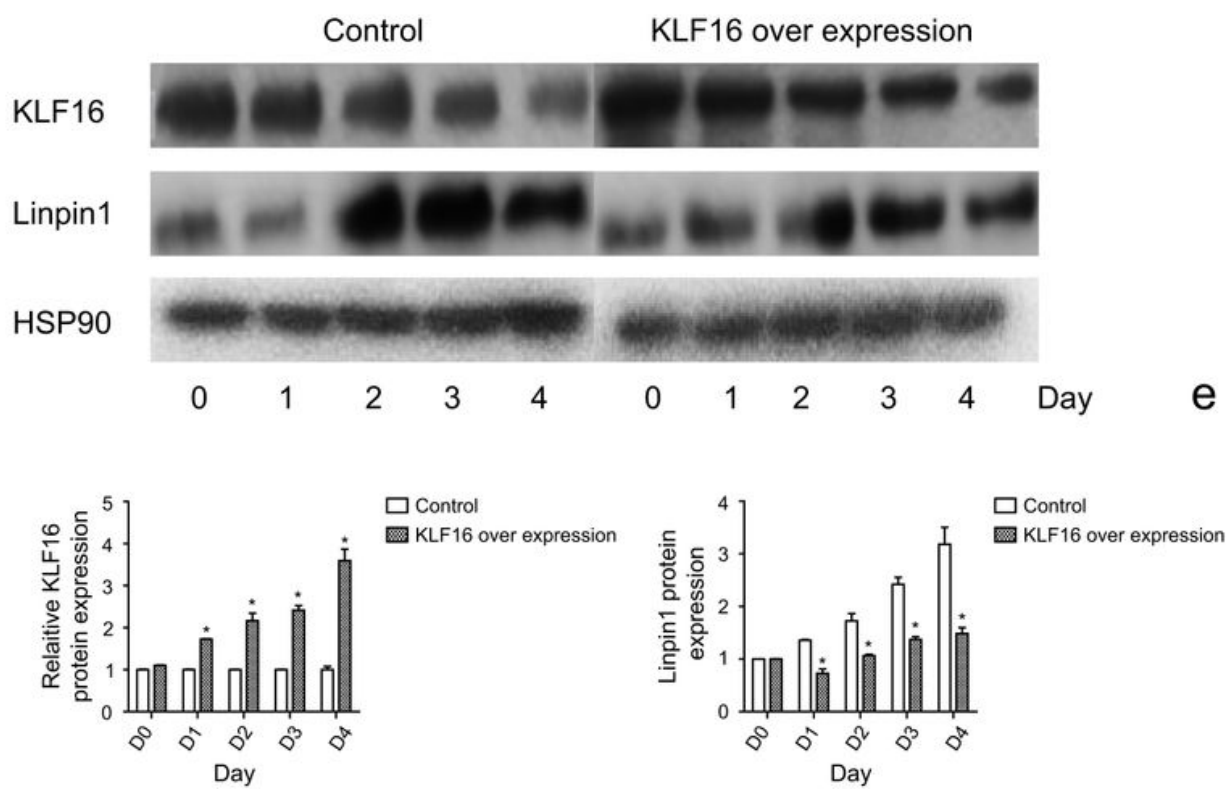

b

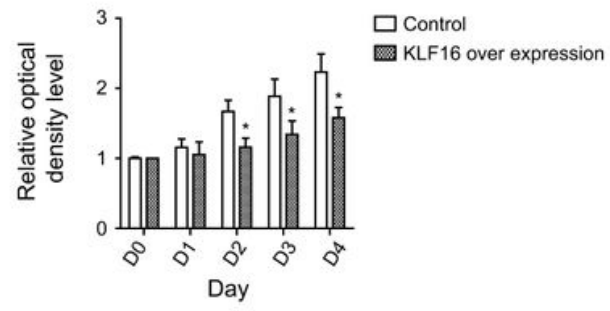

C
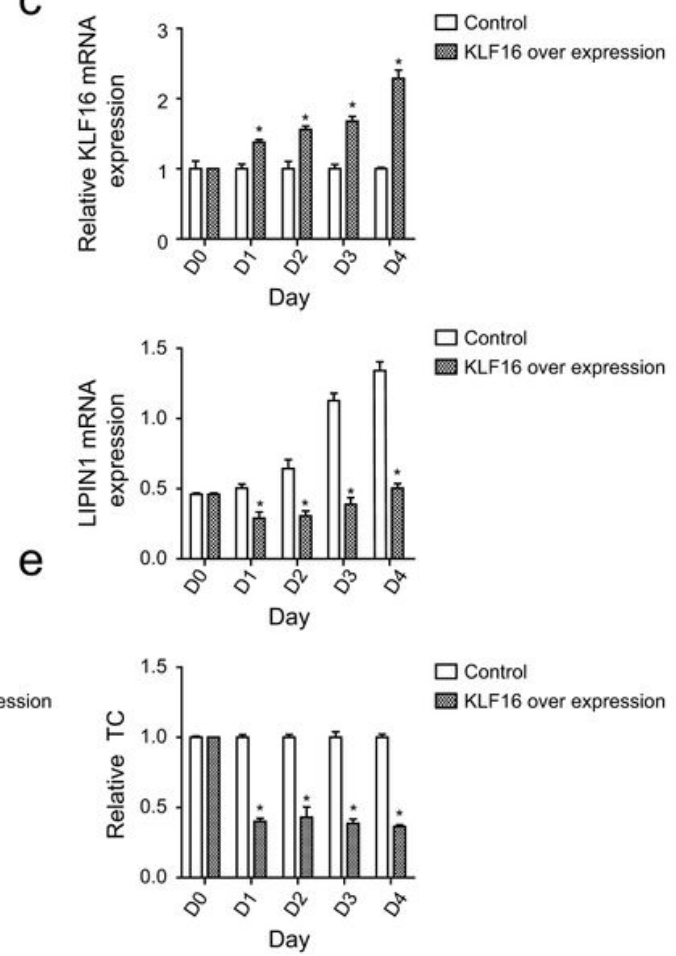

$\square$ Control

KLF16 over expression

Figure 5

KIf16 regulates LIPIN1 to affect adipocyte differentiation Representative Oil Red 0 staining images of differentiation-induced adipocytes transfected with a KLF16-overexpression plasmid for days 1, 2, 3, and 4 (a-b). Effects of KLF16 overexpression on the mRNA (c) and protein levels (d) of KLF16 and LIPIN1 compared to the control groups on days $0,1,2,3$, and 4 of 3T3-L1 adipocyte differentiation. The triglyceride content in 3T3-L1 cells of the control group and the KLF16 overexpression group was assayed using a triglyceride kit (e) 